\title{
Effects of Poly(vinyl alcohol) on Fiber Cement Interfaces. Part II: Microstructures
}

\author{
T-J. Chu and R.E. Robertson \\ Macromolecular Science and Engineering Center and Department of Materials Science and \\ Engineering, The University of Michigan, Ann Arbor, Michigan \\ H. Najm and A.E. Naaman \\ Department of Civil and Environmental Engineering, The University of Michigan, \\ Ann Arbor, Michigan
}

Changes in the microstructure of hydrated cement paste around steel, brass, brass-coated steel, and polypropylene fibers induced by the addition of $1.4 \%$ poly(vinyl alcohol) (PVA) have been examined by scanning electron microscopy. The microstructures were examined both after the fibers had been carefully peeled from the matrix and after the fibers had been pulled from the matrix along their axes. The previously noted enhancement by PVA of the pull-out properties from ordinary Portland cement matrix of steel fibers is attributed to the formation of a ductile, fine-grained interfacial layer. The formation of this microstructure is suggested to arise from the effect of PVA on the nucleation of $\mathrm{CH}$ and $\mathrm{C}-\mathrm{S}-\mathrm{H}$ at the fiber surface and on the presence of polymer around the fibers. The enhancement of the pull-out properties for brass and brass-coated steel fibers is attributed to the formation of a strong bond between the fiber and matrix that diverts failure to the porous zone surrounding the fiber. Interfacial structures against low surface energy fibers, like polypropylene, showed minimal effect from the polymer. ADVANCED Cement Based Materials 1994, 1, 122-130

KEY WORDS: Adhesion, Bond, Bond strength, Brass fibers, Cement matrix, Debonding, Friction, Poly(vinyl alcohol), Pull-out work, Slip, Steel fibers

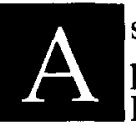
described in the accompanying article [1], poly(vinyl alcohol) (PVA) added to ordinary Portland cement (OPC) is capable of enhancing the bond properties between the cement and fibers. With 1.4\% PVA, the bond strength and frictional resistance were approximately doubled for steel fibers embedded in the cement. The postpeak load-drop was not as drastic as with unmodified cement, and the pullout work was more than doubled. Though not as effective as with plain steel fibers, PVA also enhanced

Address correspondence to: Richard E. Robertson, Department of Materials Science and Engineering, The University of Michigan, Ann Arbor, Michigan 48109$2 \uparrow 36$. the pull-out characteristics of brass or brass-coated steel fibers. The properties of a fiber composite are sensitive to the interfacial structure between the reinforcement and the matrix. This leads to the question of how had the interfacial microstructure been modified by PVA.

The interfacial zones adjacent to the fiber in OPC matrices have been studied for various fibers that have included steel [2,3], glass [4], and polypropylene [5]. The interfacial structures were found to be substantially different from those in the bulk paste [6]. The typical interfacial microstructure against steel fiber has been described to be the following [7]: Adjacent to the interface is a thin "duplex" film consisting first of a calcium hydroxide $(\mathrm{CH})$ layer and then a calcium silicate hydrate $(\mathrm{C}-\mathrm{S}-\mathrm{H})$ layer. Between this duplex layer and the bulk is a porous zone consisting of clusters of $\mathrm{C}-\mathrm{S}-\mathrm{H}$ gel, some ettringite fibrils, and large $\mathrm{CH}$ crystals in various orientations. Both the interfacial and porous regions constitute weaknesses in the structure.

The purpose of this article is to describe the interfacial structure formation in the presence of PVA and how this modified structure enhances the mechanical properties.

\section{Experimental}

The fibers studied were of plain steel, brass-coated steel, and polypropylene monofilament. These were precleaned by rubbing the surfaces with an acetonesoaked, lint-free tissue. For the matrix, a type I OPC was used. The PVA used was Airvol-203 (Air Products and Chemicals, Inc.), which is $87-89 \%$ hydrolyzed poly(vinyl acetate) in granular form. Its molar mass was relatively low, 7000-13,000 number average, $13,000-23,000$ weight average. 
The OPC was blended with water in a mixer at a water-to-cement ratio of 0.35 . For the PVA-modified specimens, the water was replaced by a $4 \%$ solution of PVA in water. The solution was made by adding small portions of PVA powder at a time into preheated $(60$ $70^{\circ} \mathrm{C}$ ) water with constant stirring. This process was continued until all of the PVA was dissolved. At the same water-to-cement ratio of 0.35 , the final amount of PVA in the matrix, based on weight of OPC in the matrix, was $1.4 \%$.

Two types of specimens were made. In the first, the fibers were embedded in the matrix for pull-out testing; in the second, the fibers were embedded for observation of the structure before pull-out. For the pullout test specimens, a block of matrix 1 in. $(25.4 \mathrm{~mm})$ thick was used. Four fibers were placed vertically in each block, about 2 in. $(50.8 \mathrm{~mm})$ apart, with the fibers extending above the top surface but not extending through the bottom surface. Further details of the pullout specimens are described in the accompanying article [1]. For specimens made to observe the structure before pull-out, the fibers were placed horizontally near the bottom of the mold, as shown in Figure 1. Specimens with and without PVA were prepared in the same ways. The specimens were cast into plastic molds, and all specimens were vibrated to reduce air entrainment.

The pull-out specimens were set and demolded after 1 day and cured in water for 1 week. They were then sealed in a large plastic bag to continue the exposure to a high humidity environment for a total of 28 to 30 days, after which the pull-out tests were performed. The second set of specimens, for observing the structure before pull-out, were sealed for 28 days in containers of nearly saturated humidity, obtained by equilibration with wet absorbent paper.

After demolding and overnight vacuum drying of the specimens, the fibers were peeled away from the matrix with great care, leaving grooves of mainly undisturbed matrix at the fiber interface. The fiber and matrix surfaces were examined by scanning electron microscopy (Hitachi S-800). Micrographs of the matrix were taken of both the top (as molded; bottom, when

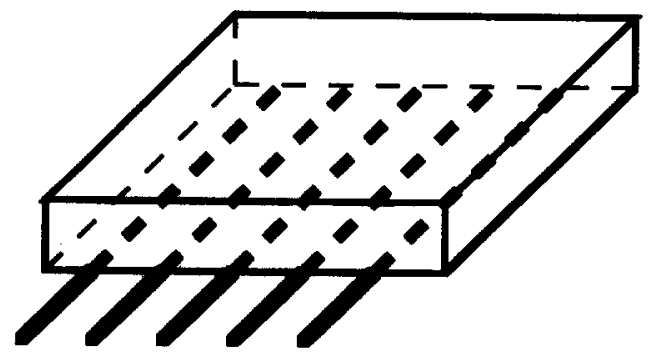

FIGURE 1. Locations of fibers in the mold for fiber-peeling experiments. turned over) and sides of the groove, as indicated in Figure 2. Stereo pairs were used to examine the threedimensional topography.

Interfaces subjected to fiber pull-out were exposed by fracturing those specimens through the cylindrical holes where the fibers had been. The holes were essentially broken in half, exposing the grooves, and the surface and edges of the grooves were examined by scanning electron microscopy.

\section{Results}

\section{Steel Fibers}

The matrix interface with the steel fibers before pullout and without PVA is shown in Figure 3. Most of the interfacial surface consists of a thin duplex layer of $\mathrm{CH}$ (adjacent to the steel) and $\mathrm{C}-\mathrm{S}-\mathrm{H}$ and containing scattered perforations. Through the perforations can be seen the fibrous porous layer beneath the surface that separates the duplex layer from the bulk. In addition, there are several large $\mathrm{CH}$ plates obliquely oriented to the surface. The corresponding fiber surface is shown in Figure 4. Matrix hydration products dot about $60-70 \%$ of the fiber surface. These had been pulled from the duplex layer and sometimes from the fibrous porous layer below. On the other hand, clean regions on the fiber duplicated smooth regions of the matrix surface. Thus, elongated regions up to $50 \mu \mathrm{m}$ in length that are free of hydration products and constitute the other $30-40 \%$ of the fiber surface were found to match the smooth, nonperforated regions of the duplex layer and $\mathrm{CH}$ plates on the matrix.

After the fiber has been pulled out from the matrix along its axis, the matrix (still without PVA) adjacent to the steel fiber appeared to have been crumbled by the pull-out process (see Figure 5). The fiber after pull-out is nearly clean with relatively little debris adhering to it.

The PVA-modified matrix at the interface with the steel fibers before pull-out is shown in Figure 6. The

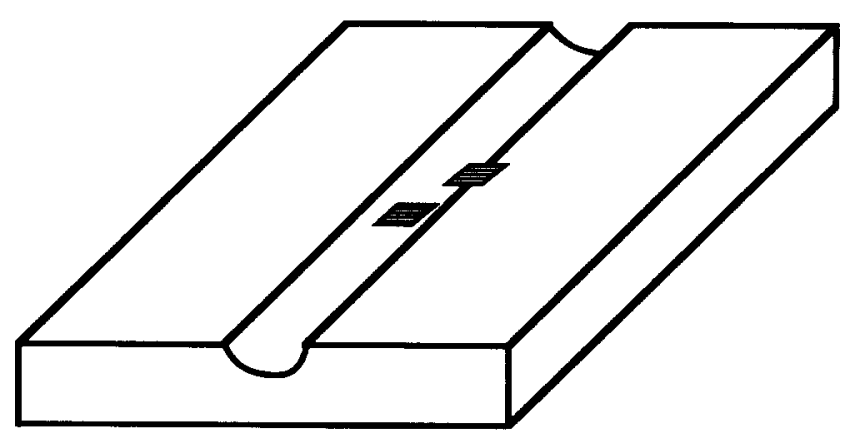

FIGURE 2. The groove after fiber peeled away, and the locations of scanning electron micrographs. 


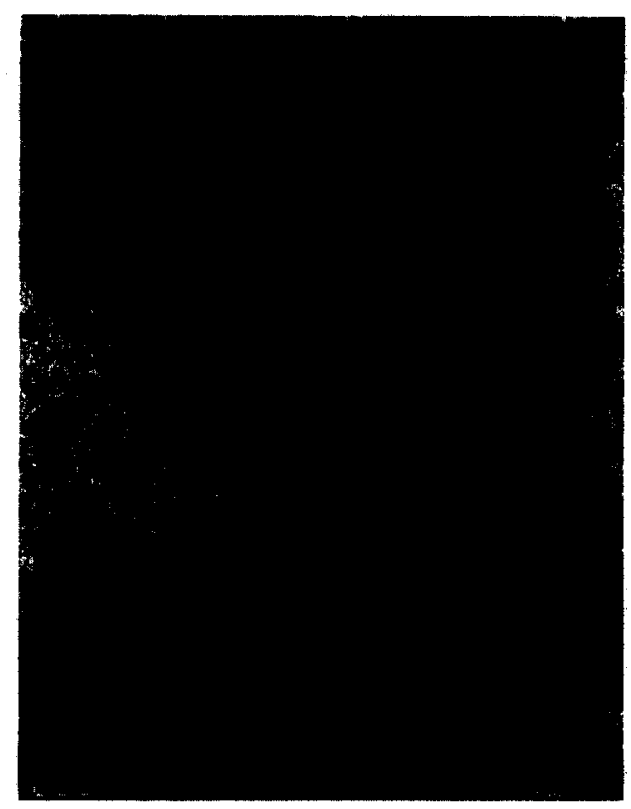

FIGURE 3. Surface of matrix without PVA after removal of steel fiber by peeling.

interface appeared to lack the continuous duplex layer over most of the surface, but the surface still replicates the contour of the steel fiber, on which there were fissures that ran roughly along the fiber axis. An edgeview of the groove is shown in Figure 7. A relatively compact layer of crystals and PVA roughly $1 \mu \mathrm{m}$ thick exists at the interface. Beneath this is a somewhat looser packing of material that is generally denser than the fibrous porous layer obtained without PVA. (Large

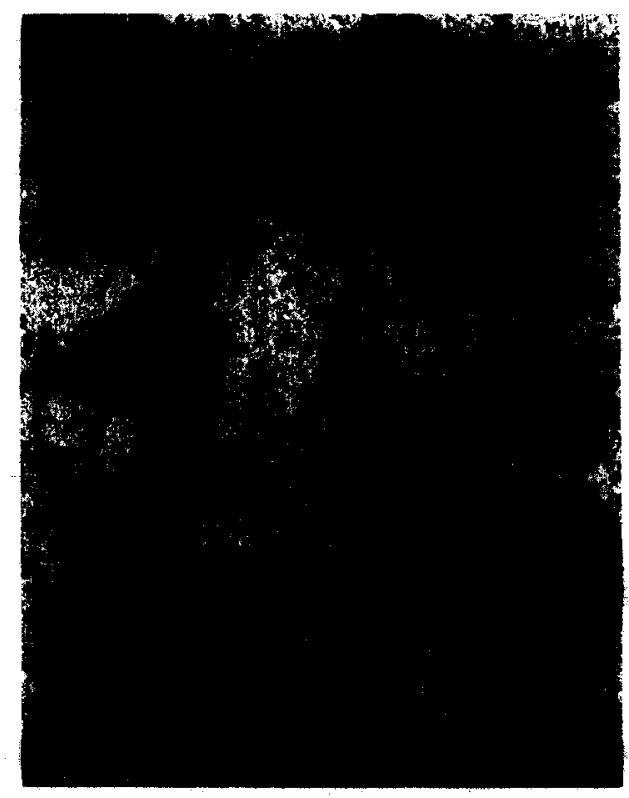

FIGURE 4. Surface of steel fiber after removal from matrix without PVA by peeling.

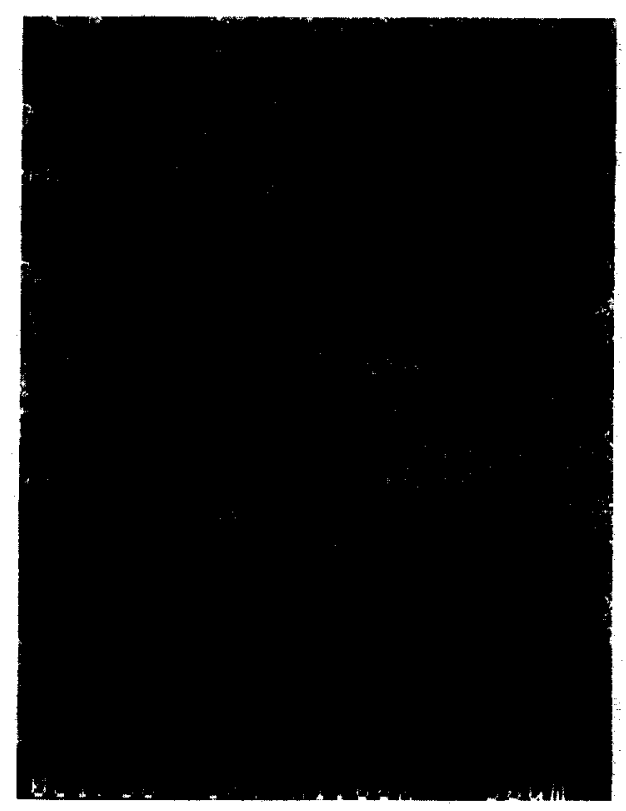

FIGURE 5. Surface of matrix without PVA after removal of steel fiber by pull-out.

pores like that on the right-hand side of Figure 7 are relatively uncommon; this pore was included in the micrograph to show that they do occasionally occur.)

After the fiber had been pulled out from the PVAmodified matrix, the matrix in the vicinity of the interface with steel fibers was found to have a smooth, dense, and fine-grained structure (see Figure 8). Though this layer contains surface cracks perpendicular to the pull-out direction, it appears to have behaved

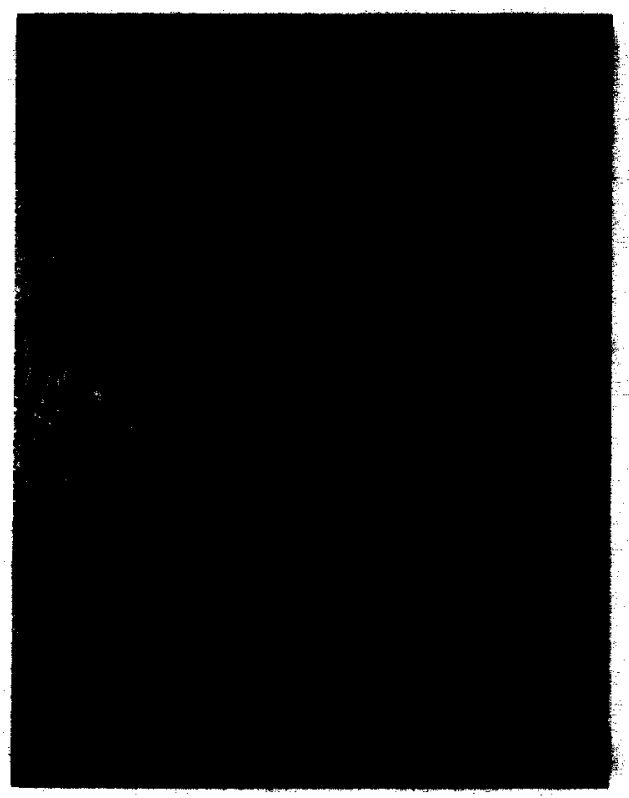

FIGURE 6. Surface of matrix with PVA after removal of steel fiber by peeling. 


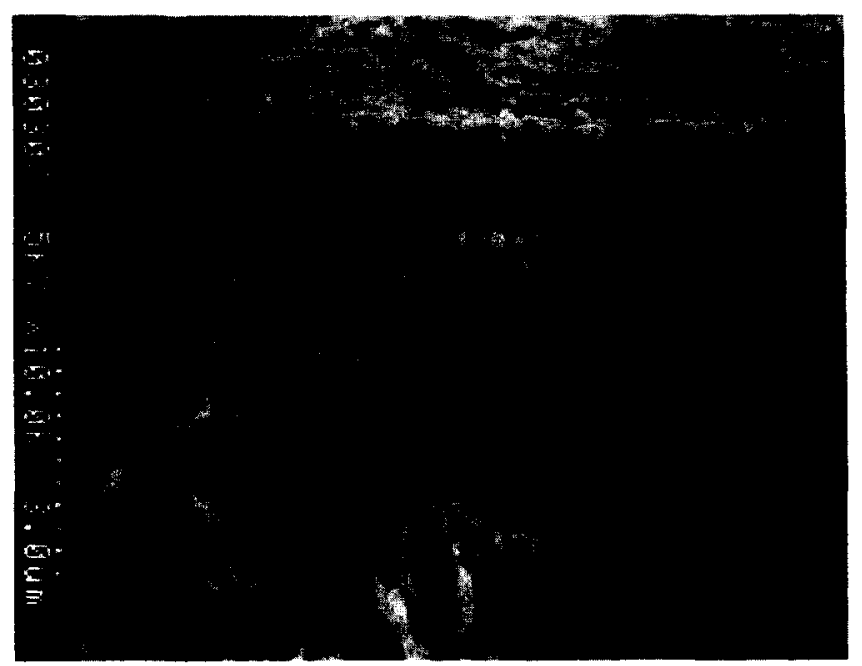

FIGURE 7. Edge-view of matrix with PVA at the interface with a steel fiber after the fiber had been removed by peeling.

in a largely ductile manner during pull-out, which has resulted in longitudinal corrugations being inscribed in the surface (see also Figures 7 to 11 in ref 1). That the superficial cracks are perpendicular to the pull-out direction suggests that they were caused by the fiber pull-out. They may have been further opened by shrinkage during drying of the specimen in preparation for scanning electron microscopy, however. A side-view of the interfacial zone is shown in Figure 9. The loose structure existing around the fiber before pull-out has been compacted, and the $1 \mu \mathrm{m}$-thick denser surface layer has merged with the less dense material beneath it. The entire surface layer is likely to be an inorganic/PVA composite.

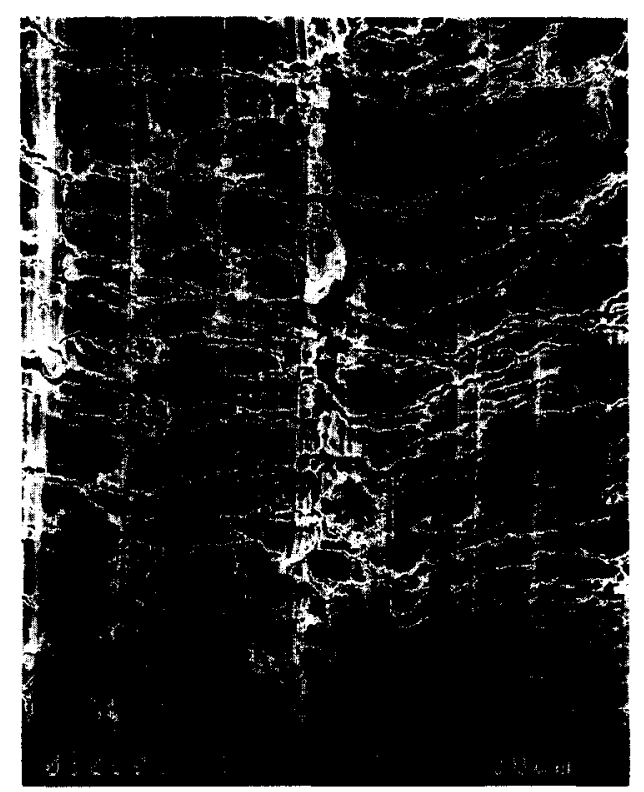

FICURE 8. Surface of matrix with PVA after removal of steel fiber by pull-out.

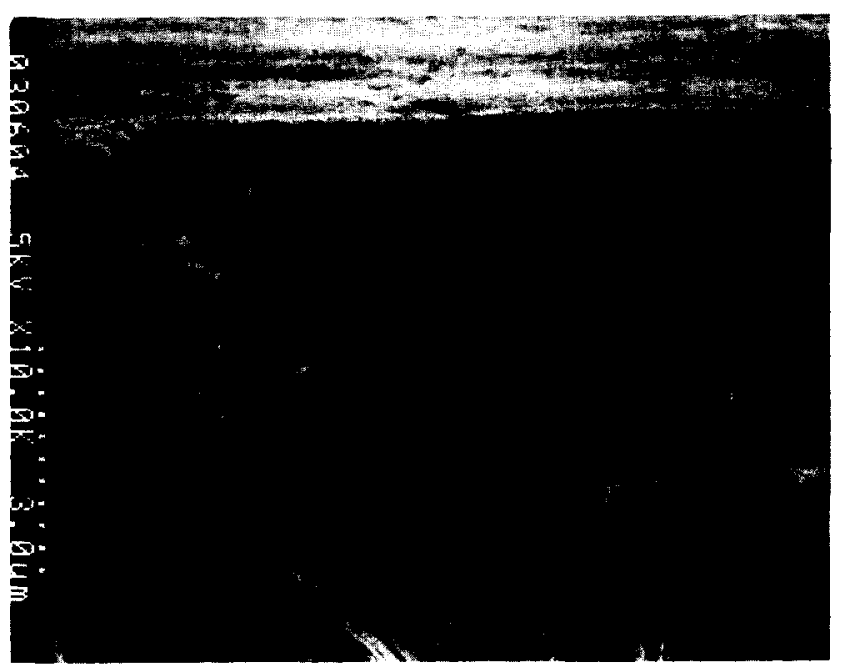

FIGURE 9. Edge-view of matrix with PVA at the interface with a steel fiber after the fiber had been removed by pullout.

\section{Brass and Brass-Coated Steel Fibers}

The surface of the matrix without PVA after carefully peeling away a brass-coated steel fiber is shown in Figure 10. The surface is rough because a thin layer of material right at the interface remained bonded to the fiber. The matrix surface in Figure 10 consists largely of several micrometer-thick plates; the plates themselves appear at higher magnification to consist of closely packed columns of material oriented perpendicular to the surface. Beneath the plates is a fibrous porous zone. After the fibers had been pulled out along their

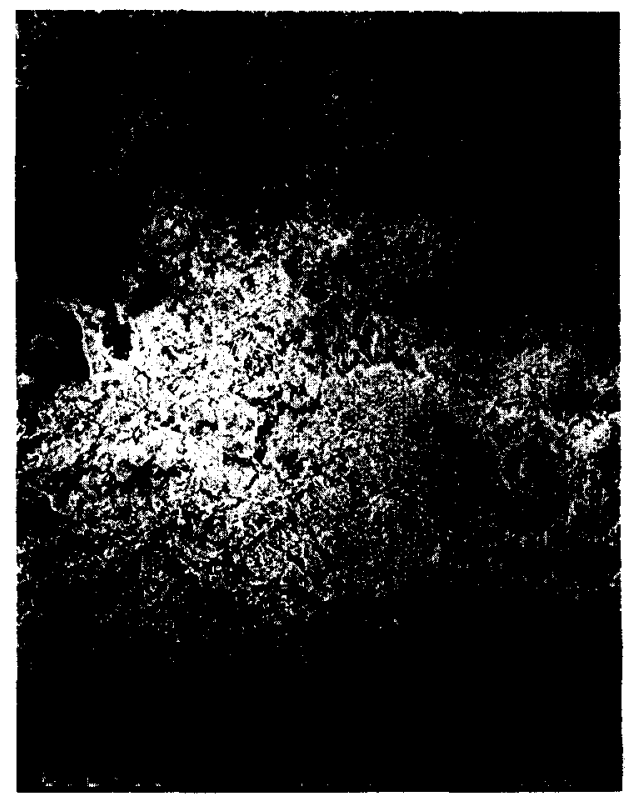

FIGURE 10. Surface of matrix without PVA after removal of brass-coated steel fiber by peeling. 
axes from the matrix without PVA, the plates at the surface of the matrix were found to have been broken into pieces, the fibrous porous layer beneath had been crumbled, and the two layers had become mixed (see Figure 11).

The surface of the matrix with PVA after carefully peeling away a brass-coated steel fiber is shown in Figure 12. The structure of the matrix is similar to that without PVA except that the surface plates are smaller and are less dense, with space between the plates. In addition, the interfacial zone of the matrix appeared to the naked eye to be greenish, suggesting a distribution of $\mathrm{Cu}^{+2}$ ions in the matrix. The surface of the fiber that had been peeled away from the matrix is shown in Figure 13. More material remains adhered to this fiber than to the fiber peeled from the matrix without PVA.

After the fibers had been pulled out along their axes from the matrix with PVA, the matrix was heavily damaged with both large and small debris (see Figure 14). Even the bulk appears to have become involved in pull-out. The corresponding fiber surface was covered with a thick layer of hydration products, as well as debris.

\section{Polypropylene Fibers}

The matrix surface without PVA after peeling away a polypropylene fiber is shown in Figure 15. The surface is quite smooth with a few cracks, possibly arising from drying in preparation for scanning electron microscopy. Also visible are striations replicated from the

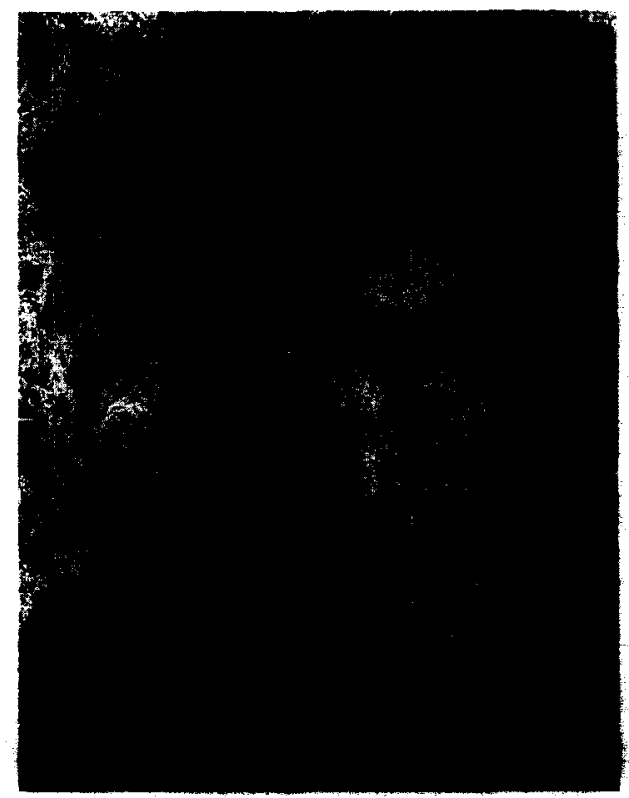

FIGURE 11. Surface of matrix without PVA after removal of brass fiber by pull-out.

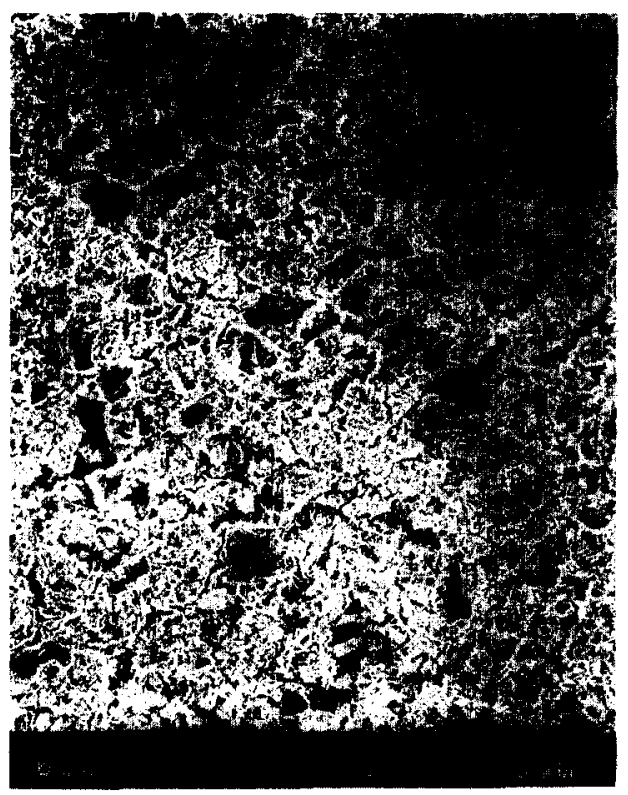

FICURE 12. Surface of matrix with PVA after removal of brass-coated steel fiber by peeling.

fiber. At higher magnification, the material at the surface was found not to be especially dense. The entire surface appeared to be filled with hydration products growing out from the matrix, rather than nucleating at the fiber surface. After pull-out, the matrix remained undamaged, maintaining a smooth texture. The force required to pull the polypropylene fiber from the matrix was very low, about $1 \mathrm{lb}(5 \mathrm{~N})$.

The surface of the PVA-modified matrix before pull-

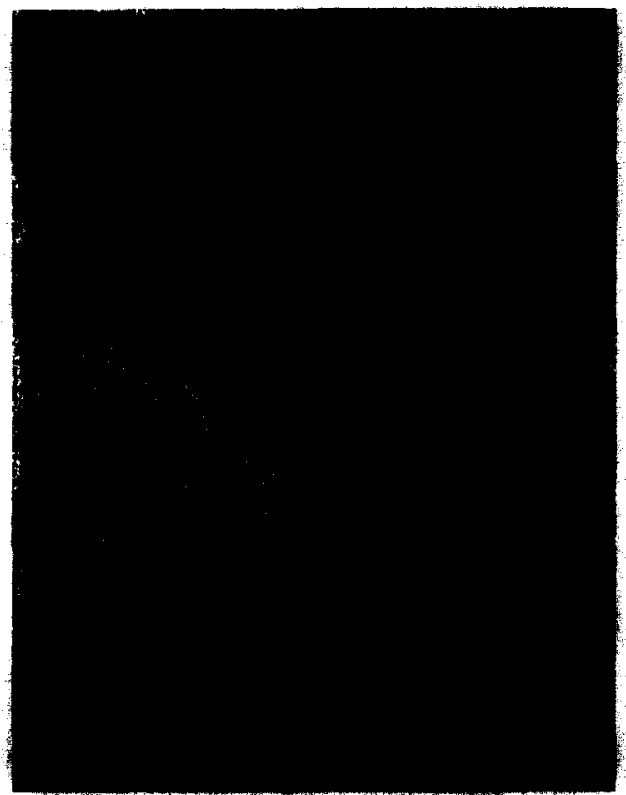

FIGURE 13. Surface of brass-coated steel fiber after removal from matrix with PVA by peeling. 


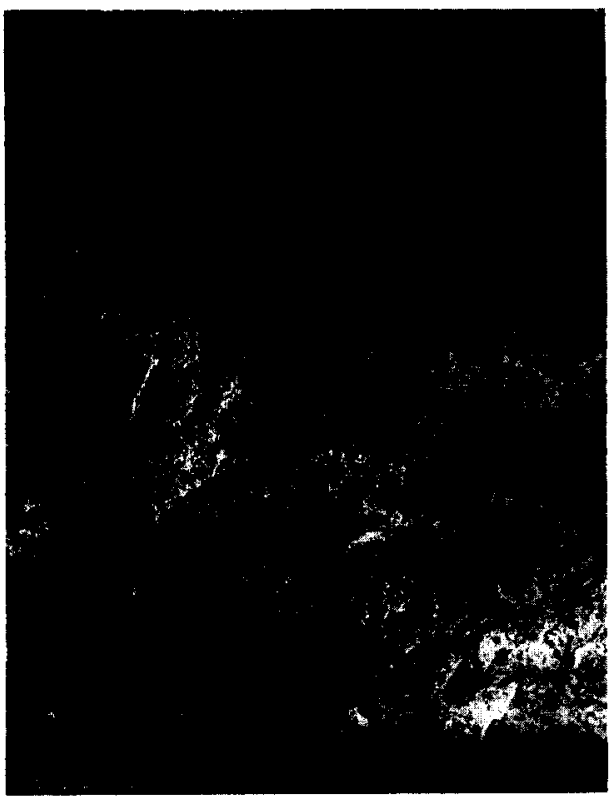

FIGURE 14. Surface of matrix with PVA after removal of brass fiber by pull-out.

out is shown in Figure 16. The surface layer is less dense than without PVA and is again mainly composed of hydration products (probably most $\mathrm{C}-\mathrm{S}-\mathrm{H}$ ) that appeared to grow against the fiber from the bulk. After fiber pull-out, the matrix surface remained essentially the same, with perhaps some of the pores having been closed by fiber slippage. Again, the pull-out force was very low, about $1 \mathrm{lb}(5 \mathrm{~N})$.

A summary of the various microstructures in the

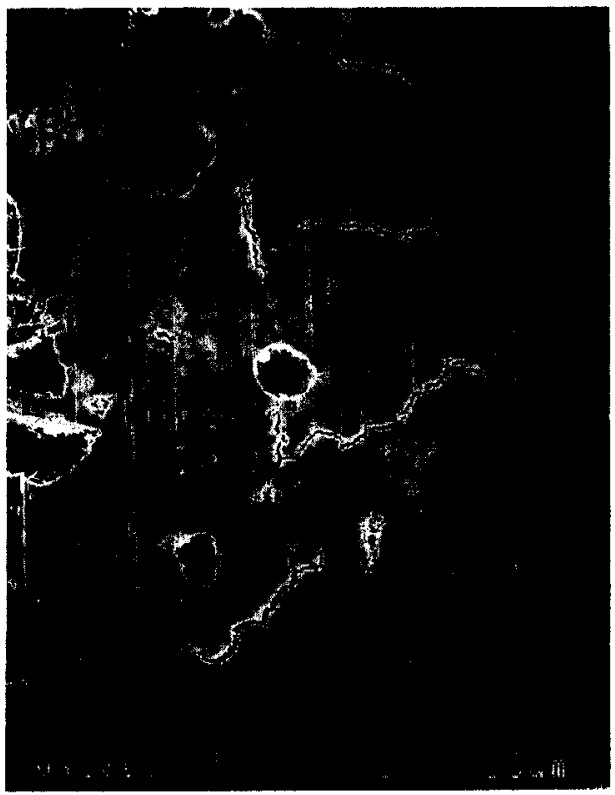

FIGURE 15. Surface of matrix without PVA after removal of polypropylene fiber by peeling.

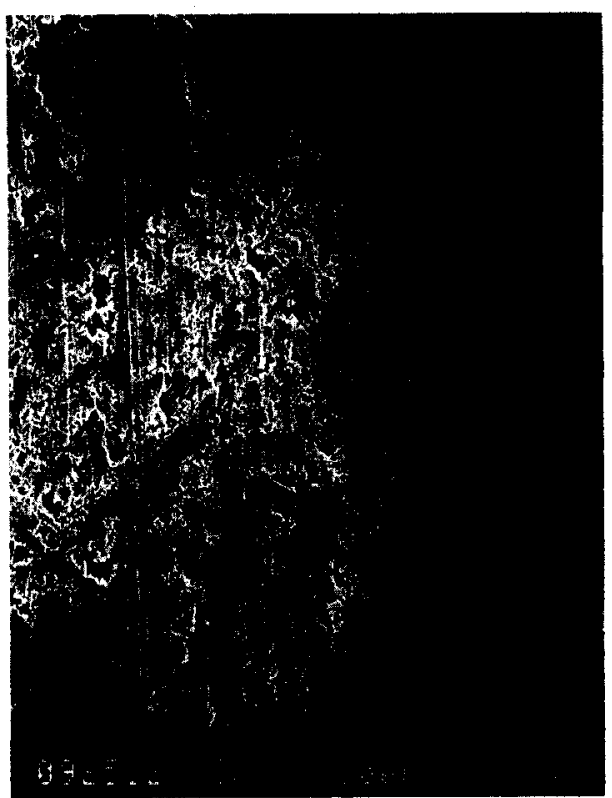

FIGURE 16. Surface of matrix with PVA after removal of polypropylene fiber by peeling.

matrix with and without PVA seen at the interface with steel, brass, and polypropylene fibers is given in Table 1 .

\section{Discussion}

With steel fibers, the addition of PVA to OPC matrix was found to cause the formation of a dense, finegrained, and ductile interfacial layer that lacked a continuous duplex layer at the interface. The porous layer remained but was reduced in porosity. With brass and brass-coated steel fibers, the addition of PVA to the OPC matrix was found to increase the adhesion at the interface; a layer of hydration products became firmly attached to the fiber surface. With polypropylene fibers, the addition of PVA to the OPC matrix was found to have little effect on the structure at the interface. In addition, little damage to the matrix at the interface was found after pull-out.

\section{Steel Fibers}

The dense, fine-grained, and ductile interfacial layer at the steel fibers is probably the result of both changes in the inorganic materials at the interface and the presence of bulk polymer. Except for the polypropylene fibers, the fiber surfaces can nucleate the deposition of hydration products, namely, $\mathrm{CH}$ crystals and perhaps $\mathrm{C}-\mathrm{S}-\mathrm{H}$. Thus, $\mathrm{CH}$ crystals of the typical duplex layer are nucleated on steel fiber surfaces, and the $\mathrm{C}-\mathrm{S}-\mathrm{H}$ is deposited on the $\mathrm{CH}$ [7]. When coated with a thin layer of lubricating oil, however, steel fibers exhibited 
TABLE 1. Interfacial structures of fiber-reinforced, PVA-modified OPC

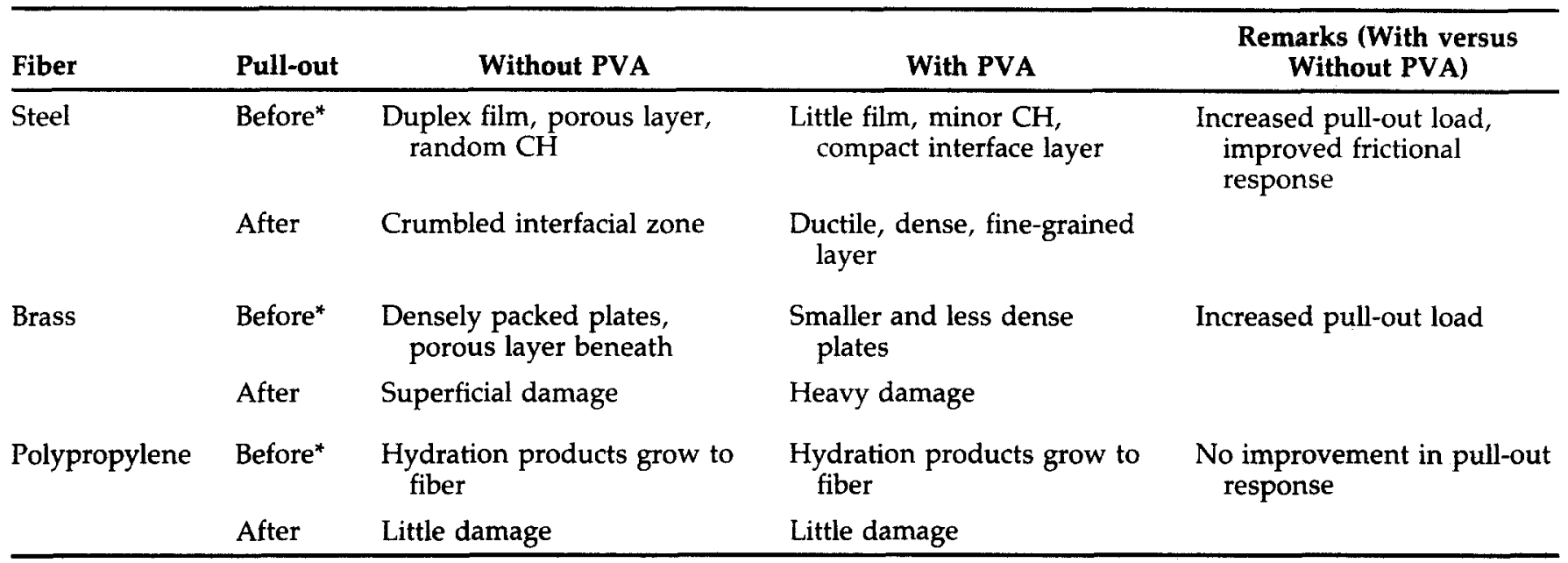

*Interfaces were exposed before pull-out by careful peeling.

the same inability to nucleate hydration products as did polypropylene fibers [8]. The presence of the PVA in the PVA-modified matrix leads to adsorption of PVA molecules on the fiber surfaces and to a similar change in nucleation, though not nearly as much as does the layer of oil.

PVA is molecularly dispersed in the water, and each PVA molecule can become attached to a hydrophilic surface, like that of steel, when only one of its many hydroxyl groups bonds to the surface. In general, when the molecule is close enough to be bonded at all, more than one hydroxyl group will be bonded to the surface. Though these bonds are in a dynamic equilibrium of forming and breaking, not unless all of the bonds are broken simultaneously is the PVA molecule free of the surface. The result is an increased concentration of PVA molecules in the water near hydrophilic surfaces, and the polymer may even coat the surface with a monomolecular film. A recent study [9] has found that PVA tends to inhibit the nucleation of $\mathrm{CH}$ and to enhance the formation of $\mathrm{C}-\mathrm{S}-\mathrm{H}$ at hydrophilic surfaces during the hydration of tricalcium silicate $\left(C_{3} S\right)$. This is expected to have happened in the present experiments. The hydration products that were observed to have formed in the presence of PVA were notable both for the relative absence of large $\mathrm{CH}$ crystals and for the presence of a fine-grained $\mathrm{C}-\mathrm{S}-\mathrm{H}$ microstructure.

The polymer is also expected to affect the porous layer surrounding the fibers. The porous layer arises from the inefficient packing of cement particles around the fibers, and a water-rich zone is created. By inhibiting the nucleation of $\mathrm{CH}$ crystals on the steel-fiber interface, the $\mathrm{CH}$ is expected to nucleate and grow on material in the porous layer, where it adds to this layer's density. In addition, PVA can accumulate in this layer.
The amount of PVA in this layer can be estimated as follows. The porous zone extends roughly $50 \mu \mathrm{m}$ into the bulk from the fiber. Within this zone, the greatest porosity exists over a distance of about $10-20 \mu \mathrm{m}$, and only about one-third of this volume is occupied by hydration products, the rest of the space being filled with water. Because the volume of inorganic material after hydration is approximately $30 \%$ greater than that of the original cement, the volume fraction of cement before hydration in the $10-20 \mu \mathrm{m}$ zone of maximum porosity would have been about $25 \%(=33 \% / 1.3)$. At an average water-to-cement ratio of 0.35 by weight, the ratio by volume is about one to one; but in the porous layer, the local water-to-cement ratio by volume would be about three to one. Thus, when the water is replaced by a PVA solution, about three times the amount of water (before hydration) and three times the amount of PVA will reside in the porous region as in the bulk. By adding a 4\% PVA solution to the dry cement instead of water, keeping the solution-tocement ratio at 0.35 , the resulting volume fraction of PVA in the porous layer would be about $3 \%(=4 \% \times$ $75 \%$ ). This is in addition to the $33 \%$ of the volume occupied by inorganic hydration products. Therefore, the PVA constitutes about $10 \%$ of the visible hydration products around the fiber.

As described in the accompanying article [1], the addition to an OPC matrix of $1.4 \%$ PVA, based on weight of cement, approximately doubled the bond strength to steel fibers and more than doubled the frictional resistance and the pull-out work. The postpeak drop in load was also less drastic for the PVA-modified material than without PVA. These mechanical property enhancements are attributed to the presence of the ductile, fine-grained interfacial layer. The ductility of the interfacial layer allowed a stable release of stress along the debonding front and reduced the postpeak 
load drop. As the slippage continued, the interfacial layer became denser and was able to maintain higher postpeak pull-out loads.

The degree of ductility of the interfacial matrix layer is indicated by the pattern of surface cracking seen in Figure 8 . The cracks seen are mostly perpendicular to the direction of fiber pull-out; there are very few in other directions. As described above, this layer is estimated to be about 10\% PVA by volume, the rest being inorganic hydration products. The ductility observed is greater than would be expected from a composite consisting of $90 \%$ hard particulate filler, $10 \%$ polymer. This suggests that the composite gains some of its ductility from the inorganic phase. The PVA has probably acted also to nucleate $\mathrm{C}-\mathrm{S}-\mathrm{H}$ particles, and these particles may have incorporated some of the PVA to attain a degree of ductility. The very fine-grain texture of the interfacial layer seen in Figures 8 and 9 and the change from that in Figure 7 indicates the degree to which the inorganic phase has accommodated the moving fiber as it was being pulled out.

This mechanism for improvement with PVA is different from the improvement with a dispersion of acrylic polymer particles found by Wei et al. [10], for example. The improvement with the latter arose from the small size of the acrylic polymer particles, 50 to 500 $\mathrm{nm}$, that allowed them to fill in the porous region surrounding the fibers. By affecting the nucleation of hydration products, the PVA is able to be much more efficient than the acrylic particles in increasing the fiber bond strength and pull-out resistance.

\section{Brass and Brass-Coated Steel Fibers}

For brass and brass-coated steel fibers, the presence of PVA reduced the size and density of the closely packed $\mathrm{CH}$ plates nucleated at the fiber surface, again probably because of an alteration of the nucleation sites by the adsorption of PVA. What was different between the brass and steel fibers was the reaction between the brass and PVA, as evidenced by the green coloration of the surface layers and by the firm adherence of these layers to the brass surface. The PVA is likely to have formed a complex with the copper in the surface (as well as copper ions in solution), to form a strong bond. This bonding caused the interfacial layer of the cement to remain attached to the fiber both during the attempt to peel the fiber from the matrix and during fiber pullout.

The improvements in peak pull-out load, frictional resistance, and pull-out energy by adding PVA to the matrix are less with brass and brass-coated steel fibers than with steel fibers, though the improvements are still in the range of $50 \%$. The decrease in the postpeak load drop also was not as great with brass and brasscoated steel fibers as with plain steel fibers. But the addition of PVA to the matrix has caused the mechanism of failure to change. The strong bond between the fiber and matrix has caused the site of failure to move away from the fiber surface to the porous region. The porous region is probably brittle by comparison with the ductile interfacial layer with steel fibers, so that the overall response of the brass-fiber-reinforced PVA-modified cement is more brittlelike, tending toward premature failures. It is possible that the complex that forms between copper (leached from the brass) and PVA prevents the PVA and any part of the hydration products around the fiber from being very ductile.

\section{Polypropylene Fibers}

The formation of the hydration products around the polypropylene fibers was very different from that of the metal fibers. The hydration products appeared not to have been nucleated at the fiber surface. This was consistent with the results obtained in the presence of PVA. PVA was found to alter nucleation of hydration products at the steel and brass surfaces. But because little nucleation of hydration products occurs at the polypropylene surface, there were no sites to be altered by PVA adsorption, and no alteration of the cement surface adjacent to the fiber was seen.

After pull-out, the matrix remained undamaged, maintaining a smooth texture. The force required to pull the polypropylene fiber from the matrix was very low, about $1 \mathrm{lb}(5 \mathrm{~N})$. Pulling stretched the fiber and had probably shrunk its diameter, allowing the fiber to pull away from the matrix at the debonding front. The friction then remained low as the fiber was pulled from the hole because the fiber was smaller than the hole. These hypotheses are supported by a calculation of the transverse strain and the general smoothness of the fiber and hole. The load of $5 \mathrm{~N}$ acting on a fiber of diameter $250 \mu \mathrm{m}$, modulus $5 \mathrm{GPa}$, and Poisson's ratio 0.4 (the latter two quantities are typical of polypropylene), gives a transverse shrinkage of $2 \mu \mathrm{m}$. Scanning electron microscopy examination of the fiber indicates a roughness amplitude in the circumferential direction of about $0.5 \mu \mathrm{m}$ and less than that in the longitudinal direction. In general, the matrix tends to replicate the fiber. Hence, the fiber would have been able to be pulled from the hole with a minimum of frictional resistance.

\section{Conclusions}

The conclusions of this research are as follows.

1. The modification of the cement interfacial structure and bonding by PVA depends on the fiber substrate. 
2. With steel fibers, the addition of PVA to OPC matrix caused the formation of a dense, finegrained, and ductile interfacial layer in place of the usual continuous duplex layer.

3. The significant increases in maximum pull-out force and frictional resistance with steel fibers in a PVA-modified matrix is suggested to arise from the strength and durability of the dense, fine-grained, and ductile PVA/cement composite.

4. With brass and brass-coated steel fibers, the addition of PVA to OPC matrix led to a chemical reaction between the PVA and the copper in the brass and the formation of a strong bond.

5. Only modest increases in bond strength and pull-out work occurred with brass and brasscoated steel fibers in PVA-modified cement because the layer surrounding the fibers, lacking ductility, had diverted failure to the still fairly weak porous zone.

6. The effect of PVA was minimal with fibers having low energy or hydrophobic surfaces like polypropylene, due to the lack of nucleation sites on these surfaces.

\section{Acknowledgments}

We acknowledge the very helpful discussions with Professor Sidney Diamond, Purdue University. This study was supported by the NSF Center for Science and Technology for Advanced Cement Based Materials (ACBM), NSF grant no. DMR-8808432.

\section{References}

1. Najm, H.; Naaman, A.E.; Chu, T.-J.; Robertson, R.E. J. Adv. Cement Based Mater. 1994, 1, 115-121.

2. Al Khalaf, M.N.; Page, C.L. Cem. Conc. Res. 1979, 9, 197.

3. Pinchin, D.J.; Tabor, D. Cem. Conc. Res. 1978, 8, 15.

4. Bentur, A. In Bonding in Cementitious Composites, Vol. 114; Mindess, S.; Shah, S.P., Eds.; Materials Research Society, Pittsburgh, PA, 1988; p 133.

5. Rice, E.K.; Vondran, G.L.; Kunbargi, H.O. In Bonding in Cementitious Composites, Vol. 114; Mindess, S.; Shah, S.P., Eds.; Materials Research Society, Pittsburgh, PA, $1988 ; \mathrm{p} 145$.

6. Bentur, A.; Mindess, S. Fiber Reinforced Cementitious Composites; Elsevier, New York, 1990; p 22.

7. Bentur, A.; Diamond, S.; Mindess, S. J. Mater. Sci. 1985, 20,3610 .

8. Chu, T.-J.; Robertson, R.E., to be published.

9. Kim, J.K.; Robertson, R.E.; Chu, T.-J. J. Mater. Sci., to be published.

10. Wei, S.; Mandel, J.A.; Said, S. ACI J. 1986, 83, 597-605. 